\title{
Breast cancer worry in higher-risk women offered preventive therapy: a UK multicentre prospective study
}

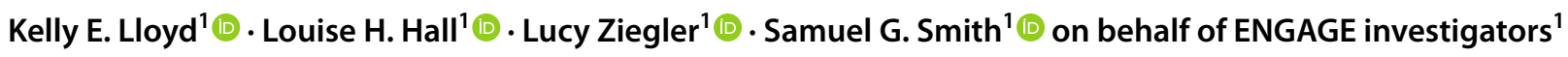

Received: 19 October 2020 / Accepted: 5 March 2021 / Published online: 17 March 2021

(c) The Author(s) 2021

\begin{abstract}
Purpose Women's worry about developing breast cancer may influence their decision to use preventive therapy. However, the direction of this relationship has been questioned. We prospectively investigated the relationship between breast cancer worry and uptake of preventive therapy. The socio-demographic and clinical factors associated with high breast cancer worry were also investigated.

Methods Women at increased risk of developing breast cancer were recruited from clinics across England $(n=408)$. Participants completed a survey on their breast cancer worry, socio-demographic and clinical factors. Uptake of tamoxifen was recorded at 3 months $(n=258$ women, $63.2 \%)$. Both primary and sensitivity analyses were conducted using different classifications of low, medium and high worry.

Results $39.5 \%$ of respondents reported medium breast cancer worry at baseline and $21.2 \%$ reported high worry. Ethnic minority women were more likely to report high worry than white women $(\mathrm{OR}=3.02,95 \% \mathrm{CI} 1.02,8.91, p=0.046)$. Women educated below degree level were more likely to report high worry than those with higher education $(\mathrm{OR}=2.29,95 \%$ CI 1.28 , $4.09, p=0.005$ ). No statistically significant association was observed between worry and uptake. In the primary analysis, fewer respondents with medium worry at baseline initiated tamoxifen (low worry $=15.5 \%$, medium $=13.5 \%$, high $=15.7 \%$ ). In the sensitivity analysis, participants with medium worry reported the highest uptake of tamoxifen (19.7\%).

Conclusions No association was observed between worry and uptake, although the relationship was affected by the categorisation of worry. Standardised reporting of the classification of worry is warranted to allow transparent comparisons across cohorts.
\end{abstract}

Keywords Preventive therapy $\cdot$ Chemoprevention $\cdot$ Decision-making $\cdot$ Cancer worry

\section{Introduction}

Breast cancer incidence is rising worldwide [1]. There is increasing interest in preventive therapy for people at higher risk of cancer as part of prevention efforts [2,3]. The UK National Institute of Health and Care Excellence (NICE) considers women with a lifetime breast cancer risk of $17-30 \%$ to be at moderately high risk, and those exceeding $30 \%$ to be high risk $[4,5]$. In a meta-analysis of nine randomised control trials, selective oestrogen receptor modulators (SERMs) reduced the incidence of breast cancer by

Samuel G. Smith

s.smith1@leeds.ac.uk

1 Leeds Institute of Health Sciences, University of Leeds, Worsley Building, Clarendon Way, Leeds LS2 9NL, UK at least 30\% among women at increased risk [6]. In 2013, NICE published clinical guidelines (CG164) recommending SERMs (e.g. tamoxifen) are offered to higher-risk women with a family history of breast cancer [4].

Following the introduction of the NICE guidance (CG164), a UK prospective study reported low uptake of tamoxifen (14.7\%) among women at increased risk of breast cancer [7]. Worry about developing breast cancer may influence women's decision to use preventive therapy. Previous cross-sectional studies found women reporting higher levels of breast cancer worry were more likely to be interested in using preventive therapy $[8,9]$. Prospective US studies have also observed an association between breast cancer worry and uptake of preventive therapy $[10,11]$. These findings suggest women who worry more about developing breast cancer may be more likely to use preventive therapy. 
However, there are mixed results on the direction of this relationship [12].

There may be an inverted U-shaped relationship between breast cancer worry and uptake of preventive therapy, whereby moderate levels of worry are most strongly associated with uptake. An inverted U-shaped relationship has been observed between breast cancer worry and other cancer protective behaviours, such as screening [13-16]. These findings suggest people with moderate worries about developing breast cancer are more likely to engage with preventive behaviours, compared with those reporting lower or higher worries. Few prospective studies have investigated whether there is a U-shaped relationship between breast cancer worry and uptake of preventive therapy. Given the negative psychological side effects of worry [17], identifying those who experience cancer-related worry is warranted. Previous data also indicate people from lower socioeconomic status (SES) backgrounds [18], and ethnic minority respondents $[18,19]$ are more likely to experience higher levels of cancer worry.

We assessed levels of breast cancer worry among women considering preventive therapy in a UK clinical practice setting and investigated whether there was an association between worry and uptake. We also investigated the sociodemographic and clinical factors associated with higher worry, and assessed whether worry changed over time. This analysis is part of the ENGAGE study; a multicentre prospective investigation of preventive therapy uptake in England among women at increased risk of breast cancer [7, 20].

\section{Methods}

\section{Recruitment}

Twenty clinics in England supported recruitment between September 2015 and December 2016. This included family history clinics $(n=12)$, breast clinics $(n=4)$, clinical genetic centres $(n=3)$ and a family history clinic with genetics support $(n=1)$. After a clinic appointment, a healthcare professional approached the woman to discuss the study. Women who verbally consented were given a study pack containing the baseline survey. Informed consent was implied following the return of the survey. An identification code was assigned to participants who verbally consented, which was linked to their baseline survey. After verbal consent, women's personal data were sent to the study team via a secure online portal. These data included: patients age, email address, home address and their risk classification ('moderately high' or 'high'). At 3 months, baseline survey respondents were sent a follow-up survey.

The ENGAGE study was granted ethical approval by the National Research Ethics Service Committee North
West—Preston (14/NW/1408). Women were eligible to participate if they were over the age of 18 , classified as 'moderately high' or 'high' risk of breast cancer, spoke English, had discussed preventive therapy with a healthcare professional and had no known contraindications for tamoxifen use. Women were excluded if they were unable to consent or had a previous breast cancer diagnosis.

\section{Measures}

The baseline survey contained measures of socio-demographic and clinical factors, and breast cancer worry. The follow-up survey measured uptake of tamoxifen and breast cancer worry at 3 months. The time period between the baseline and follow-up survey was decided on the basis that this was a reasonable amount of time for participants to consider the harms and benefits of tamoxifen, and to have spoken with a GP about obtaining a prescription.

\section{Socio-demographic and clinical factors}

The baseline survey contained items on demographic and socioeconomic factors, some of which were dichotomised in the analysis. These measures included nulliparity (yes vs. no), marital status (married/cohabiting vs. single/ divorced/separated/widowed), education level ( $\geq$ degree level vs. $<$ degree level), ethnicity (white ethnic groups vs. ethnic minority groups), employment status (full-time vs. all other employment types) and self-reported health (poor, fair, good, excellent). Age was calculated from date of birth provided from National Health Service (NHS) records, with age recoded to $\leq 35$ years; $36-49$ years and; $\geq 50$ years. Postcodes were used to calculate a participant's Index of Multiple Deprivation (IMD) score, and women were classified into tertiles of neighbourhood deprivation (low, middle and high) [21]. Breast cancer risk category, based on the NICE guideline CG164 [4], was provided by staff at the clinic they had attended.

\section{Breast cancer worry}

Two items measured breast cancer worry, which were adapted from the Lerman Breast Cancer Worry Scale [16]. Respondents were asked to assess how frequently each statement was true for them over the past 7 days. The statements were 'I worried about developing breast cancer' and 'My worries about breast cancer interfered with my daily activities'. Each item was assessed on a 4-point Likert scale: 'Not at all', 'Rarely', 'Sometimes' and 'Often'. Women were classified as reporting low/medium/high worry using a method adapted from Zhang et al. [14]. Participants reporting 'Not at all' or 'Rarely' to both worry questions were classified as low worry. Participants reporting 'Sometimes' to either or 
both questions were classified as medium worry, and participants reporting 'Often' to either or both questions were classified as high worry. The breast cancer worry scale was administered to participants at baseline and 3-month followup. The Cronbach's alpha for the scale was 0.73 .

\section{Preventive therapy decision}

At 3 months, we measured self-reported uptake of tamoxifen. Women were asked to tick which of the following categories applied to them: (1) 'I decided immediately that I did not want to take tamoxifen'; (2) 'After some thought, I decided that I did not want to take tamoxifen'; (3) 'I am still deciding if I want to take tamoxifen'; (4) 'I met with my GP to talk about tamoxifen, and I decided against it'; (5) 'I met with my GP to talk about tamoxifen, but they would not prescribe it'; (6) 'I have a prescription for tamoxifen from my GP'; (7) 'I am currently taking tamoxifen'. Women were considered to be using tamoxifen if they ticked categories 6 or 7, with responses 1-5 indicating no uptake.

\section{Data analysis}

The socio-demographic and clinical factors were compared between responders and non-responders to the baseline questionnaire, and responders and non-responders to the 3-month questionnaire using Pearson's Chi-square test. Responses on the baseline worry scale were compared between responders and non-responders to the 3-month questionnaire using a between-subjects $t$ test.

We used Pearson's Chi-square test to assess for differences in baseline breast cancer worry ('low/medium' vs. 'high') across each of the socio-demographic and clinical factors. Fisher's exact test was used if $>20 \%$ of cells had an expected count $<5$. We conducted a multivariable logistic regression model, including all socio-demographic and clinical variables to identify factors associated with high breast cancer worry at baseline (vs. 'low/medium' worry).

Univariable and multivariable logistic regression models examined whether women's baseline breast cancer worry ('low', 'medium' or 'high') was associated with uptake of tamoxifen at 3 months. The multivariable model was adjusted for all socio-demographic and clinical factors. We used a McNemar test to assess for differences in baseline and 3 -month worry across the three different worry groups. We used a within-subjects $t$ test to compare mean worry scores between baseline and 3-month follow-up.

Sensitivity analyses were conducted to assess if the approach used to classify the worry groups affected the study's findings [22]. In the sensitivity analysis, participants reporting 'Not at all' to both worry questions were classified as low worry. Participants reporting 'Rarely' to either or both worry questions were classified as medium worry, and participants reporting 'Sometimes' or 'Often' to either or both worry questions were classified as high worry.

The analysis plan for the study was pre-registered (https:// osf.io/6dupm). The analysis was conducted in SPSS version 25 , with statistical significance set at a 2 -sided $p<0.05$.

\section{Results}

In total, 732 women consented to participate. For the baseline survey, 408 (55.7\%) women responded, and 258 (63.2\%) women returned the 3-month follow-up survey. Among the 408 baseline respondents, $275(67.4 \%)$ attended a family history clinic, $49(12.0 \%)$ attended a breast clinic, $25(6.1 \%)$ attended a clinical genetic centre, and 59 (14.5\%) attended a family history clinic with genetics support. Participant characteristics are presented in Table 1.

There were no significant differences between responders to the baseline survey (408 women) and non-responders (324 women) with regard to clinical risk $(p=0.62)$, socioeconomic status $(p=0.054)$ or age $(p=0.086)$. Baseline respondents retained at 3 months were more likely to be from a higher socioeconomic status (SES) group $(p<0.001)$. There were no significant differences in the mean baseline worry scores between responders who only responded to the baseline survey compared with those who completed both the baseline and 3-month survey $(p=0.234)$.

\section{Breast cancer worry and participant characteristics}

In total, 408 women provided baseline worry data. Among these, 39.3\% had low baseline breast cancer worry, 39.5\% had moderate worry, and $21.2 \%$ had high worry. For each of the worry items, $21.2 \%$ worried about developing breast cancer 'often', while only $3.5 \%$ felt their worries about breast cancer 'often' interfered with their daily activities (Table 1).

In a multivariable logistic regression model (Table 2), women from an ethnic minority group were more likely to report high breast cancer worry than white women $(\mathrm{OR}=3.02,95 \% \mathrm{CI} 1.02,8.91, p=0.046)$. Women who were educated to below degree level were more likely to report high worry than women educated to degree level or above $(\mathrm{OR}=2.29,95 \%$ CI $1.28,4.09, p=0.005)$.

We carried out a sensitivity analysis whereby participants who responded 'Sometimes' or 'Often' to either or both worry questions were categorised into the high worry group. In the sensitivity analysis, $52(12.8 \%)$ women were categorised as having low worry at baseline. Most women (246; 60.7\%) were categorised as having high worry, and 107 (26.4\%) were categorised as moderate worry (Supplementary Table 1S). Ethnicity was no longer significantly associated with high worry in the sensitivity analysis (Table 2S); however, the effect size with education was 
Table 1 Description of participants demographic, clinical and breast cancer worry characteristics at baseline $(n=408)$

\begin{tabular}{|c|c|}
\hline Demographic, clinical and worry factors & Baseline respondents \\
\hline Age, mean \pm SD & $45.30 \pm 7.82$ \\
\hline \multicolumn{2}{|l|}{ Age, $n(\%)$} \\
\hline$\leq 35$ years & $41(10)$ \\
\hline 36-49 years & $259(63.5)$ \\
\hline$\geq 50$ years & $108(26.5)$ \\
\hline \multicolumn{2}{|l|}{ Children, $n(\%)$} \\
\hline Yes & $314(77.0)$ \\
\hline No & $94(23.0)$ \\
\hline \multicolumn{2}{|l|}{ Ethnic group, $n(\%)$} \\
\hline White ethnic groups & $384(95.5)$ \\
\hline Ethnic minority groups & $18(4.5)$ \\
\hline Caribbean & 3 \\
\hline Indian & 5 \\
\hline Pakistani & 2 \\
\hline Other Asian & 1 \\
\hline White and Black Caribbean & 2 \\
\hline White and Black African & 1 \\
\hline Other mixed ethnicity & 2 \\
\hline Any other & 2 \\
\hline Missing, $n$ & 6 \\
\hline \multicolumn{2}{|l|}{ Education level, $n(\%)$} \\
\hline Degree or above & $176(44.2)$ \\
\hline Below degree level & $222(55.8)$ \\
\hline Missing, $n$ & 10 \\
\hline \multicolumn{2}{|l|}{ Health status, $n(\%)$} \\
\hline Poor & $16(4.0)$ \\
\hline Fair & $78(19.5)$ \\
\hline Good & $240(60.0)$ \\
\hline Excellent & $66(16.5)$ \\
\hline Missing, $n$ & 8 \\
\hline \multicolumn{2}{|l|}{ Risk level, $n(\%)$} \\
\hline Moderate & $243(59.6)$ \\
\hline High & $159(39.0)$ \\
\hline Unclear & $6(1.5)$ \\
\hline \multicolumn{2}{|l|}{ SES, $n(\%)$} \\
\hline Low (most deprived) & $120(29.9)$ \\
\hline Middle & $131(32.7)$ \\
\hline High (least deprived) & $150(37.4)$ \\
\hline Missing, $n$ & 7 \\
\hline \multicolumn{2}{|l|}{ Employment, $n(\%)$} \\
\hline Full-time & $348(85.3)$ \\
\hline All other employments & $60(14.7)$ \\
\hline \multicolumn{2}{|l|}{ Marital status, $n(\%)$} \\
\hline Married or cohabiting & $298(74.3)$ \\
\hline Unmarried & $103(25.7)$ \\
\hline Missing, $n$ & 7 \\
\hline \multicolumn{2}{|l|}{ Breast cancer worry, $n(\%)$} \\
\hline Low worry & $159(39.3)$ \\
\hline Medium worry & $160(39.5)$ \\
\hline
\end{tabular}

Table 1 (continued)

\begin{tabular}{ll}
\hline Demographic, clinical and worry factors & Baseline respondents \\
\hline High worry & $86(21.2)$ \\
Missing, $n$ & 3 \\
Worry about developing breast cancer, $n(\%)$ & \\
Not at all & $52(12.8)$ \\
Rarely & $108(26.7)$ \\
Sometimes & $159(39.3)$ \\
Often & $86(21.2)$ \\
Missing, $n$ & 3 \\
Worries interfered with daily activities, $n(\%)$ & \\
Not at all & $267(65.9)$ \\
Rarely & $84(20.7)$ \\
Sometimes & $40(9.9)$ \\
Often & $14(3.5)$ \\
Missing, $n$ & 3 \\
\hline
\end{tabular}

SES socioeconomic status, $S D$ standard deviation

similar $(\mathrm{OR}=2.00,95 \%$ CI 1.27, 3.16, $p=0.003)$. In addition, women with poor/fair $(\mathrm{OR}=2.90,95 \% \mathrm{CI} 1.43,5.92$, $p=0.003)$ and good health status (OR $=1.80,95 \%$ CI 1.00 , $3.24, p=0.050$ ) were more likely to report high breast cancer worry than those with excellent health status.

\section{Uptake of preventive therapy and breast cancer worry}

Among the 258 women who completed the 3-month follow-up questionnaire, 38 (14.7\%) had initiated tamoxifen. A higher proportion of women in the low worry group $(16 / 103$; $15.5 \%)$ and high worry group $(8 / 51 ; 15.7 \%)$ at baseline initiated preventive therapy, compared with the medium worry group $(14 / 104 ; 13.5 \%)$. A multivariable logistic regression model examined the association of breast cancer worry and uptake, after adjusting for all socio-demographic and clinical variables. Breast cancer worry was not significantly associated with tamoxifen initiation in the multivariable model (Table 3). No socio-demographic factors were significantly associated with uptake in the multivariable model (Table 3).

In the sensitivity analysis, we found that a higher proportion of women categorised as medium worry at baseline $(13 / 66 ; 19.7 \%)$ initiated tamoxifen, compared with women categorised as high worry $(22 / 155 ; 14.2 \%)$ and low worry $(3 / 37 ; 8.1 \%)$. None of the worry levels were significantly associated with tamoxifen uptake (Table 3S).

\section{Breast cancer worry at baseline and 3 months}

Breast cancer worry was compared between baseline and 3 months in the sample of women who provided both baseline and follow-up data (Table 4). A higher proportion of 
Table 2 Breast cancer worry by participant characteristics and Pearson's Chi-square test and multivariable logistic regression model $(n=385)$

\begin{tabular}{|c|c|c|c|c|c|}
\hline \multicolumn{2}{|c|}{ High breast cancer worry $(n ; \%)$} & \multicolumn{2}{|c|}{ Chi-square } & \multicolumn{2}{|l|}{ Multivariable } \\
\hline & & \multirow{2}{*}{$\begin{array}{l}X^{2} \\
1.21\end{array}$} & \multirow{2}{*}{$\begin{array}{l}P \text { value } \\
0.546\end{array}$} & \multirow[t]{2}{*}{ OR $(95 \% \mathrm{CI})$} & \multirow[t]{2}{*}{$P$ value } \\
\hline Age & & & & & \\
\hline$\leq 35$ years & $6(14.6)$ & & & $0.58(0.17-2.02)$ & 0.393 \\
\hline $36-49$ years & $57(22.2)$ & & & $1.40(0.77-2.60)$ & 0.273 \\
\hline$\geq 50$ years & $23(21.5)$ & & & Ref & \\
\hline Children & & 0.20 & 0.656 & & \\
\hline Yes & $68(21.7)$ & & & $1.11(0.56-2.21)$ & 0.773 \\
\hline No & $18(19.6)$ & & & Ref & \\
\hline Ethnic group & & 6.28 & 0.032* & & \\
\hline White & $76(19.8)$ & & & Ref & \\
\hline Ethnic minority & $8(44.4)$ & & & $3.02(1.02-8.91)$ & 0.046 \\
\hline Education level & & 9.61 & 0.002 & & \\
\hline Degree or above & $25(14.2)$ & & & Ref & \\
\hline Below degree level & $60(27.0)$ & & & $2.29(1.28-4.09)$ & 0.005 \\
\hline Health status & & 3.18 & 0.204 & & \\
\hline Poor/fair & $25(26.6)$ & & & $1.43(0.58-3.51)$ & 0.434 \\
\hline Good & $49(20.4)$ & & & $1.13(0.50-2.53)$ & 0.767 \\
\hline Excellent & $10(15.2)$ & & & Ref & \\
\hline Risk level & & 2.93 & $0.219 *$ & & \\
\hline Moderate & 45 (18.7) & & & Ref & \\
\hline High & $39(24.7)$ & & & $1.24(0.72-2.11)$ & 0.440 \\
\hline Unclear & $2(33.3)$ & & & $1.21(0.19-7.87)$ & 0.843 \\
\hline SES & & 7.30 & 0.026 & & \\
\hline Low (most deprived) & $32(26.9)$ & & & $1.89(0.96-3.71)$ & 0.066 \\
\hline Middle & $30(23.1)$ & & & $1.79(0.92-3.47)$ & 0.085 \\
\hline High (least deprived) & $21(14.0)$ & & & Ref & \\
\hline Employment & & 1.85 & 0.173 & & \\
\hline Full-time & $70(20.1)$ & & & Ref & \\
\hline All other employments & $16(28.1)$ & & & $1.34(0.65-2.80)$ & 0.431 \\
\hline Marital status & & 0.43 & 0.514 & & \\
\hline Married or cohabiting & $61(20.5)$ & & & $0.69(0.37-1.28)$ & 0.236 \\
\hline Unmarried & $24(23.5)$ & & & Ref & \\
\hline
\end{tabular}

Bold text indicates statistical significance $(P \leq 0.05)$

*Fisher's exact test participants were categorised as high breast cancer worry at baseline $(51 / 258 ; 19.8 \%)$, compared with the proportion categorised as high worry at the 3-month follow-up (32/248; $12.9 \%)$. A lower proportion of women were categorised as low worry at baseline $(103 / 258 ; 39.9 \%)$ compared with 3 months $(115 / 248 ; 46.4 \%)$.

There was no significant difference in the proportion of women categorised as low, medium and high breast cancer worry at baseline compared with the worry categories at 3 months $(p=0.077)$. A within-subjects comparison of the average worry level indicated a significant decline in worry between baseline $(M=4.09, \mathrm{SD}=1.54)$ and 3 months $(M=3.72, \mathrm{SD}=1.57 ; p<0.001)$.

In the sensitivity analysis, the proportion of women categorised as medium worry and high worry decreased between baseline (low worry $=14.3 \%$, medium $=25.6 \%$, high $=60.1 \%$ ) and 3-month follow-up (low worry $=29.8 \%$, medium $=16.5 \%$, high $=53.6 \% ; p<0.001)$.

\section{Discussion}

\section{Summary of findings}

In this UK prospective study of women at increased risk of breast cancer, over one-fifth of women reported high worry immediately after an appointment to discuss their cancer risk. Breast cancer worry was not consistent over time. Higher worry was reported immediately after the appointment than at 3-month follow-up, although half of 
Table 3 Uptake of tamoxifen by breast cancer worry and participant characteristics in the univariable and multivariable logistic regression model $(n=247)$

\begin{tabular}{|c|c|c|c|c|c|}
\hline \multicolumn{2}{|l|}{ Uptake of tamoxifen $(n ; \%)$} & \multicolumn{2}{|l|}{ Univariable } & \multicolumn{2}{|l|}{ Multivariable } \\
\hline & & OR $(95 \% \mathrm{CI})$ & $P$ value & OR $(95 \% \mathrm{CI})$ & $P$ value \\
\hline \multicolumn{6}{|l|}{ Breast cancer worry } \\
\hline Low worry & $16(15.5)$ & Ref & & Ref & \\
\hline Medium worry & $14(13.5)$ & $0.85(0.39-1.84)$ & 0.672 & $0.87(0.38-2.01)$ & 0.744 \\
\hline High worry & $8(15.7)$ & $1.01(0.40-2.55)$ & 0.980 & $1.07(0.38-3.05)$ & 0.899 \\
\hline \multicolumn{6}{|l|}{ Age } \\
\hline$\leq 35$ years & $1(3.8)$ & $0.28(0.03-2.36)$ & 0.242 & $0.36(0.04-3.41)$ & 0.373 \\
\hline $36-49$ years & $29(17.3)$ & $1.46(0.63-3.39)$ & 0.378 & $1.30(0.49-3.42)$ & 0.597 \\
\hline$\geq 50$ years & $8(12.5)$ & Ref & & Ref & \\
\hline \multicolumn{6}{|l|}{ Children } \\
\hline Yes & $36(17.6)$ & $5.43(1.26-23.34)$ & 0.023 & $4.66(0.99-21.99)$ & 0.052 \\
\hline No & $2(3.8)$ & Ref & & Ref & \\
\hline \multicolumn{6}{|l|}{ Ethnic group } \\
\hline White & $37(15.0)$ & $1.41(0.17-11.60)$ & 0.750 & $1.91(0.20-18.06)$ & 0.572 \\
\hline Ethnic minority & $1(11.1)$ & Ref & & Ref & \\
\hline \multicolumn{6}{|l|}{ Education level } \\
\hline Degree or above & $20(17.2)$ & $1.41(0.71-2.82)$ & 0.327 & $1.83(0.80-4.16)$ & 0.152 \\
\hline Below degree level & 18 (12.9) & Ref & & Ref & \\
\hline \multicolumn{6}{|l|}{ Health status } \\
\hline Poor/fair & $5(10.6)$ & $0.68(0.20-2.32)$ & 0.538 & $0.73(0.20-2.67)$ & 0.629 \\
\hline Good & $25(16.6)$ & $1.13(0.46-2.82)$ & 0.787 & $1.24(0.47-3.29)$ & 0.666 \\
\hline Excellent & 7 (14.9) & Ref & & Ref & \\
\hline \multicolumn{6}{|l|}{ Risk level } \\
\hline Moderate & $24(15.1)$ & $1.05(0.52-2.15)$ & 0.885 & $0.91(0.42-1.95)$ & 0.910 \\
\hline High & $14(14.4)$ & Ref & & Ref & \\
\hline Unclear & 0 & - & - & - & - \\
\hline \multicolumn{6}{|l|}{ SES } \\
\hline Low (most deprived) & 7 (11.9) & $0.78(0.30-2.03)$ & 0.613 & $1.13(0.41-3.15)$ & 0.814 \\
\hline Middle & $14(16.3)$ & $1.13(0.52-2.47)$ & 0.759 & $1.71(0.72-4.08)$ & 0.227 \\
\hline High (least deprived) & $16(14.7)$ & Ref & & Ref & \\
\hline \multicolumn{6}{|l|}{ Employment } \\
\hline Full-time & $32(14.5)$ & Ref & & Ref & \\
\hline All other employments & $6(16.2)$ & $1.14(0.44-2.96)$ & 0.783 & $1.50(0.54-4.18)$ & 0.438 \\
\hline \multicolumn{6}{|l|}{ Marital status } \\
\hline Married or cohabiting & $33(16.7)$ & $2.16(0.80-5.81)$ & 0.127 & $1.67(0.52-5.40)$ & 0.389 \\
\hline Unmarried & $5(8.5)$ & Ref & & Ref & \\
\hline
\end{tabular}

Bold text indicates statistical significance $(P \leq 0.05)$
Table 4 Proportion of women categorised as low, medium and high breast cancer worry at baseline and at 3-month follow-up, in the sample of women who provided both baseline and follow-up data $(n=258)$

\begin{tabular}{lll}
\hline & \multicolumn{3}{c}{ Baseline $(n=258)$} & 3 months $(n=248)$ \\
\hline Breast cancer worry groups, $n(\%)$ & \\
Low worry & $103(39.9)$ & $115(46.4)$ \\
Medium worry & $104(40.3)$ & $101(40.7)$ \\
High worry & $51(19.8)$ & $32(12.9)$ \\
\hline
\end{tabular}

Missing worry data for ten respondents at 3-month follow-up our sample maintained a medium or high level of worry at follow-up. Support for women considering the use of preventive therapy may be particularly needed among specific socio-demographic groups. Women from ethnic minority groups and women who had lower educational qualifications were more likely to report high breast cancer worry immediately following their appointment. 


\section{Relation to wider literature and implications for practice}

Women's worries about developing breast cancer did not appear to affect their decisions regarding tamoxifen use. These results contrast with previous prospective studies, which observed that women who had high worry about developing breast cancer were more likely to initiate preventive therapy $[10,11]$. We also investigated whether medium levels of worry were the optimal level to motivate women to initiate tamoxifen, as predicted by the C-SHIP model [23] and Yerkes-Dodson law [24]. Previous research found evidence supporting an inverted U-shaped relationship between worry and breast cancer screening attendance, whereby lower and higher worry was associated with reduced attendance [13-15]. We did not observe a U-shaped relationship between worry and uptake in the primary analysis.

There was some evidence to support the inverted U-shaped between worry and uptake in the sensitivity analysis, indicating that the interpretations of this relationship will be affected by how worry is categorised. When a higher proportion of respondents were categorised into the high worry group, the inverted U-shaped was more prominent. To assess the direction of this relationship in future research, standardised reporting of the classification of low, medium and high worry is warranted to allow transparent comparisons across cohorts. Additionally, there is a need for future studies to be pre-registered to increase the transparency of the intended analyses.

Women with lower educational qualifications, and those from ethnic minority groups were more likely to report high worry about developing breast cancer. These results are comparable to previous research that has identified similar inequalities in cancer worry in the UK general population $[18,19]$. The findings indicate inequalities in cancer worry may extend to individuals at increased risk of developing the disease. It is unclear why these groups experience greater worry about breast cancer, but previous evidence suggests some ethnic minority groups may be more fearful of the social consequences of diagnosis [25]. Further research should examine the factors influencing cancer worry in higher-risk individuals from these socio-demographic groups. Future research should also investigate which ethnic minority groups are more likely to experience higher levels of cancer worry.

Women with lower educational qualifications may need additional support when offered preventive therapy. Women educated below degree level have previously been found to report lower levels of knowledge on the benefit and harms of tamoxifen [26]. The study also found women were more likely to initiate tamoxifen if they reported greater knowledge on its benefit and harms [26]. Decision aids may be more beneficial in improving health outcomes for disadvantaged patients, compared with those with higher literacy and from higher SES backgrounds [27]. A metaanalysis of randomised control trials found strong evidence to suggest cancer-related decision aids increase patients' knowledge on the topic, without increasing anxiety [28]. Decision aids for preventive options have also been shown to reduce cancer-related distress in women at increased risk of breast cancer [29]. The development of a decision aid for preventive therapy could address knowledge and worry inequalities among women at increased risk of breast cancer.

Reducing patient's breast cancer worries is important to increase their feelings of satisfaction with treatment decisions [10]. One approach is for clinicians to adopt an 'affective communication' style, for example, being emotionally supportive with regard to patients' breast cancer-related worries when discussing their risk reducing options. An experimental study found that affective communication reduced patients' feelings of anxiety and increased their ability to recall information on treatment options [30].

Although our study did not find evidence of an association between breast cancer worry and uptake of tamoxifen, there may be other influential factors affecting uptake. Our analysis was part of the ENGAGE study; a prospective investigation of the psychological, clinical and demographic factors associated with uptake of breast cancer preventive therapy [7, 20,26]. Previous analyses of the ENGAGE cohort have observed women with children [7], and those reporting lower concerns about using tamoxifen [20] were more likely to initiate preventive therapy. Additionally, we previously found that women who felt more informed on tamoxifen were less likely to be still deciding on the medication at three month follow-up [26]. The findings from our ENGAGE study contribute to the wider literature on decision-making in the area of breast cancer preventive therapy. We also measured additional patient-reported outcomes that have not yet been reported, but have previously been found to be associated with increased uptake of preventive therapy. These include perceived risk of breast cancer [10] and intrusive (i.e. involuntary and repetitive) thoughts and feelings on breast cancer [10].

\section{Strengths and limitations}

This was the first UK study to investigate breast cancer worry and uptake of tamoxifen in a routine clinical setting. This context is important as preventive therapy uptake is higher in trial settings [31]. Women were recruited from 20 different centres and four different clinic types across England, and recruitment was open for over one year. We used a prospective design, which overcomes many of the limitations of previous cross-sectional studies assessing cancer worry $[8,9,32,33]$. 
This study had limitations. Uptake of preventive therapy was self-reported, which may have led us to overestimate this behaviour. While our sample size was reasonably large, the number of women initiating tamoxifen was low, which resulted in low precision for some comparisons. Similarly, only 18 ethnic minority participants were recruited, which reduces confidence in the associations between ethnicity, worry and uptake. Tamoxifen was the chemoprevention agent specified in the questionnaire, as the study was conducted before the introduction of anastrozole in 2017 [5]. Our findings should therefore be carefully generalised to women considering anastrozole. Additionally, we recruited women with a family history of breast cancer, and therefore our findings may not be generalisable to other potential users of tamoxifen, such as women with high-risk benign lesions.

Over $40 \%$ of women who verbally consented to participate in the study did not complete the baseline survey, which may have resulted in selection bias. While we did not find significant differences in clinical risk, socioeconomic status and age between responders and non-responders to the baseline survey, there may be other important differences in characteristics between these two groups that we did not measure (e.g. ethnicity and education level). There was also evidence of a selection bias among responders to the follow-up survey. Approximately two-thirds (63\%) of participants completed the follow-up survey at three months, with respondents from higher SES more likely to return the follow-up questionnaire. This may have caused bias in the interpretation of our data, as there is evidence to suggest cancer preventive healthcare is underutilised by those from lower SES [34, 35]. Therefore, we may have overestimated rates of uptake of preventive therapy in this cohort. Reminder postcards and questionnaires were sent to participants to minimise dropout rates in our study; however, more intensive retention approaches may be needed to improve response rates among those at lower SES [36].

\section{Conclusion}

In this UK prospective study of women at increased risk of breast cancer, most respondents reported medium to high levels of breast cancer worry following their initial appointment to discuss their cancer risk. However, worry was not linearly associated with uptake, nor was there a U-shaped association. Women with lower educational qualifications and ethnic minority women were more likely to report higher worry about developing breast cancer. Decision aids could be developed specifically for women from these socio-demographic groups, to reduce their feelings of cancer worry when offered preventive therapy. To advance the field, standardised reporting of the classification of low, medium and high worry is warranted, and pre-registered studies are required.

Supplementary Information The online version contains supplementary material available at https://doi.org/10.1007/s10549-021-06183-x.

Acknowledgements The authors acknowledge the contribution of the ENGAGE collaborators (in alphabetical order): Vanessa Adamson, Sarah Ainsworth, Malin Akerlund, Ivanna Baker, Julian Barwell, Jayne Beesley, Lisa Brock, Chrissie Butcher, Janice Carpenter, Martyn Clark, Shirley Cocks, Veronica Conteh, Martina Coulding, Sue Darby, Angela Duckworth, Gareth Evans, Catherine Fensom, Julie Fletcher, Kate Foster, Sara Grieg, Elaine Gullaksen, Jana Gurasashvili, Lisa Hardstaff, Rachel Hart, Kathryn Hoare, Jonathan Hoffman, Christopher Holcombe, Lynne Horton, Antony Howell, Farah Islam, Emma Jenkinson, Karen Jewers, Manisha Joshi, Amy Kirkby, Peter Kneeshaw, Natalie Knife, Jalal Kokan, Jin Li, Nicola Lunt, Douglas Macmillan, Karen Makinson, Evangelos Mallidis, Sarah Manyangadze, Charity Masvaure, Raksha Mistry, Alice Ngumo, Jane Ooi, Ashraf Patel, Vanessa Pope, Laura Price, Fiona Rabson, Lisa Richardson, Stephanie Ridgway, Karen Riley, Lorraine Roberts, Janet Ryan-Smith, Vian Salih, Nicky Scott, Mike Shere, Andrew Sloan, Nita Solanky, Amanda Taylor, Dinesh Thekkinkattil, Heather Thomas, Mangesh Thorat, Barbara Townley, Jayant S. Vaidya, Lynda Wagstaff, Shane Walsh, Lynsey Waring, Donna Watterson, Charlotte Westley, Lesley Wilkinson, Nicola Willis and Julia Wiseman. We also thank the women participating in the study.

Author contributions SGS designed and supervised the study. KEL analysed and interpreted the results. KEL, LHH, LZ and SGS helped to write the manuscript.

Funding Samuel G. Smith was supported by a Cancer Research UK postdoctoral fellowship (C42785/A17965) during the collection of these data. He also acknowledges funding support from a Yorkshire Cancer Research University Academic Fellowship. K. E. Lloyd is supported by an Economic and Social Research Council studentship. L. Ziegler acknowledges funding support from a Yorkshire Cancer Research University Academic Fellowship. The funder had no role in study design, data collection and analysis, decision to publish, or preparation of the manuscript.

Data availability Participants did not provide explicit consent for their data to be shared in public repositories. Therefore, data may not be made publicly available due to ethical restrictions. We can share the anonymised version of the data to individual qualified researchers upon request. Data requests may be sent to the corresponding author of this paper. The surveys used in the study and the SPSS syntax of the analysis can be found at https://osf.io/mqz9y/.

\section{Declarations}

Conflict of interest The authors declare that they have no conflict of interest.

Ethical approval Ethical approval was awarded by the National Research Ethics Service Committee North West-Preston (14/ $\mathrm{NW} / 1408$ ). All procedures performed in studies involving human participants were in accordance with the ethical standards of the institutional and/or national research committee and with the 1964 Declaration of Helsinki and its later amendments or comparable ethical standards. 
Informed consent Informed consent was obtained from all individual participants included in this study.

Open Access This article is licensed under a Creative Commons Attribution 4.0 International License, which permits use, sharing, adaptation, distribution and reproduction in any medium or format, as long as you give appropriate credit to the original author(s) and the source, provide a link to the Creative Commons licence, and indicate if changes were made. The images or other third party material in this article are included in the article's Creative Commons licence, unless indicated otherwise in a credit line to the material. If material is not included in the article's Creative Commons licence and your intended use is not permitted by statutory regulation or exceeds the permitted use, you will need to obtain permission directly from the copyright holder. To view a copy of this licence, visit http://creativecommons.org/licenses/by/4.0/.

\section{References}

1. Lin L, Yan L, Liu Y, Yuan F, Li H, Ni J (2019) Incidence and death in 29 cancer groups in 2017 and trend analysis from 1990 to 2017 from the Global Burden of Disease Study. J Hematol Oncol 12(1):96

2. Steward WP, Brown K (2013) Cancer chemoprevention: a rapidly evolving field. Br J Cancer 109(1):1-7. https://doi.org/10.1038/ bjc. 2013.280

3. Al Rabadi L, Bergan R (2017) A way forward for cancer chemoprevention: think local. Cancer Prev Res 10(1):14-35. https://doi. org/10.1158/1940-6207.CAPR-16-0194

4. National Institute for Health and Care Excellence (2013) Familial breast cancer: classification and care of people at risk of familial breast cancer and management of breast cancer and related risks in people with a family history of breast cancer. https://www.nice. org.uk/guidance/CG164. Accessed 17 Jun 2019

5. National Institute for Health and Care Excellence (2017) Familial breast cancer: classification and care of people at risk of familial breast cancer and management of breast cancer and related risks in people with a family history of breast cancer. https://www.nice. org.uk/guidance/CG164. Accessed 17 Jun 2019

6. Cuzick J, Sestak I, Bonanni B, Costantino JP, Cummings S, DeCensi A, Dowsett M, Forbes JF, Ford L, LaCroix AZ, Mershon J, Mitlak BH, Powles T, Veronesi U, Vogel V, Wickerham DL, Group SCoBCO (2013) Selective oestrogen receptor modulators in prevention of breast cancer: an updated meta-analysis of individual participant data. Lancet 381(9880):1827-1834. https://doi. org/10.1016/S0140-6736(13)60140-3

7. Hackett J, Thorneloe R, Side L, Wolf M, Horne R, Cuzick J, Smith SG (2018) Uptake of breast cancer preventive therapy in the UK: results from a multicentre prospective survey and qualitative interviews. Breast Cancer Res Treat 170(3):633-640. https://doi.org/ 10.1007/s10549-018-4775-1

8. Tjia J, Micco E, Armstrong K (2008) Interest in breast cancer chemoprevention among older women. Breast Cancer Res Treat 108(3):435-453. https://doi.org/10.1007/s10549-007-9614-8

9. Bastian LA, Lipkus IM, Kuchibhatla MN, Weng HH, Halabi S, Ryan PD, Skinner CS, Rimer BK (2001) Women's interest in chemoprevention for breast cancer. Arch Intern Med 161(13):1639-1644

10. Bober SL, Hoke LA, Duda RB, Regan MM, Tung NM (2004) Decision-making about tamoxifen in women at high risk for breast cancer: clinical and psychological factors. J Clin Oncol 22(24):4951-4957. https://doi.org/10.1200/jco.2004.05.192
11. Holmberg C, Bandos H, Fagerlin A, Bevers TB, Battaglia TA, Wickerham DL, McCaskill-Stevens WJ (2017) NRG oncology/ national surgical adjuvant breast and bowel project decisionmaking project-1 results: decision making in breast cancer risk reduction. Cancer Prev Res 10(11):625-634. https://doi.org/10. 1158/1940-6207.capr-17-0076

12. Rondanina G, Puntoni M, Severi G, Varricchio C, Zunino A, Feroce I, Bonanni B, Decensi A (2008) Psychological and clinical factors implicated in decision making about a trial of low-dose tamoxifen in hormone replacement therapy users. J Clin Oncol 26(9):1537-1543. https://doi.org/10.1200/jco.2007.13.6739

13. Andersen MR, Smith R, Meischke H, Bowen D, Urban N (2003) Breast cancer worry and mammography use by women with and without a family history in a population-based sample. Cancer Epidemiol Biomark Prev 12(4):314-320

14. Zhang LR, Chiarelli AM, Glendon G, Mirea L, Knight JA, Andrulis IL, Ritvo P (2012) Worry is good for breast cancer screening: a study of female relatives from the ontario site of the breast cancer family registry. J Cancer Epidemiol 2012:1-9. https://doi.org/10. $1155 / 2012 / 545062$

15. Sutton S, Bickler G, Sancho-Aldridge J, Saidi G (1994) Prospective study of predictors of attendance for breast screening in inner London. J Epidemiol Community Health 48(1):65-73. https://doi. org/10.1136/jech.48.1.65

16. Lerman C, Trock B, Rimer BK, Jepson C, Brody D, Boyce A (1991) Psychological side effects of breast cancer screening. Health Psychol 10(4):259-267

17. McLaughlin KA, Borkovec TD, Sibrava NJ (2007) The effects of worry and rumination on affect states and cognitive activity. Behav Ther 38(1):23-38. https://doi.org/10.1016/j.beth.2006.03. 003

18. Wardle J, McCaffery K, Nadel M, Atkin W (2004) Socioeconomic differences in cancer screening participation: comparing cognitive and psychosocial explanations. Soc Sci Med 59(2):249-261. https://doi.org/10.1016/j.socscimed.2003.10.030

19. Vrinten C, van Jaarsveld CHM, Waller J, von Wagner C, Wardle J (2014) The structure and demographic correlates of cancer fear. BMC Cancer 14(1):1-9. https://doi.org/10.1186/ 1471-2407-14-597

20. Thorneloe RJ, Horne R, Side L, Wolf MS, Smith SG et al (2019) Beliefs about medication and uptake of preventive therapy in women at increased risk of breast cancer: results from a multicenter prospective study. Clin Breast Cancer 19(1):e116-e126. https://doi.org/10.1016/j.clbc.2018.10.008

21. McLennan D, Barnes H, Noble M, Davies J, Garratt E, Dibben C (2011) The English indices of deprivation 2010. Government UK, London. https://assets.publishing.service.gov.uk/government/ uploads/system/uploads/attachment_data/file/6320/1870718.pdf. Accessed 1 Jul 2019

22. Thabane L, Mbuagbaw L, Zhang S, Samaan Z, Marcucci M, Ye C, Thabane M, Giangregorio L, Dennis B, Kosa D, Borg Debono V, Dillenburg R, Fruci V, Bawor M, Lee J, Wells G, Goldsmith CH (2013) A tutorial on sensitivity analyses in clinical trials: the what, why, when and how. BMC Med Res Methodol 13(1):1-12. https://doi.org/10.1186/1471-2288-13-92

23. Miller SM, Shoda Y, Hurley K (1996) Applying cognitive-social theory to health-protective behavior: breast self-examination in cancer screening. Psychol Bull 119(1):70-94

24. Yerkes RM, Dodson JD (1908) The relation of strength of stimulus to rapidity of habit-formation. J Comp Neurol Psychol 18(5):459-482

25. Murphy PJ, Marlow LAV, Waller J, Vrinten C (2018) What is it about a cancer diagnosis that would worry people? A populationbased survey of adults in England. BMC Cancer 18(1):86-86. https://doi.org/10.1186/s12885-017-3963-4 
26. Thorneloe RJ, Hall LH, Walter FM, Side L, Lloyd KE, Smith SG (2020) Knowledge of potential harms and benefits of tamoxifen among women considering breast cancer preventive therapy. Cancer Prev Res 13(4):411-422. https://doi.org/10.1158/1940-6207. CAPR-19-0424

27. Durand MA, Carpenter L, Dolan H, Bravo P, Mann M, Bunn F, Elwyn G (2014) Do interventions designed to support shared decision-making reduce health inequalities? A systematic review and meta-analysis. PLoS ONE 9(4):e94670. https://doi.org/10. 1371/journal.pone.0094670

28. Trikalinos TA, Wieland LS, Adam GP, Zgodic A, Ntzani EE (2014) Decision aids for cancer screening and treatment. Agency for Healthcare Research and Quality (US). https://www.ncbi.nlm. nih.gov/books/NBK269405/ Accessed 7 Aug 2019

29. Metcalfe KA, Dennis CL, Poll A, Armel S, Demsky R, Carlsson L, Nanda S, Kiss A, Narod SA (2017) Effect of decision aid for breast cancer prevention on decisional conflict in women with a BRCA1 or BRCA2 mutation: a multisite, randomized, controlled trial. Genet Med 19(3):330-336. https://doi.org/10.1038/gim. 2016.108

30. van Osch M, Sep M, van Vliet LM, van Dulmen S, Bensing JM (2014) Reducing patients' anxiety and uncertainty, and improving recall in bad news consultations. Health Psychol 33(11):13821390. https://doi.org/10.1037/hea0000097

31. Smith SG, Sestak I, Forster A, Partridge A, Side L, Wolf MS, Horne R, Wardle J, Cuzick J (2016) Factors affecting uptake and adherence to breast cancer chemoprevention: a systematic review and meta-analysis. Ann Oncol 27(4):575-590. https://doi.org/10. 1093/annonc/mdv590
32. Mandelblatt J, Kaufman E, Sheppard VB, Pomeroy J, Kavanaugh J, Canar J, Pallandre L, Cullen J, Huerta E (2005) Breast cancer prevention in community clinics: will low-income Latina patients participate in clinical trials? Prev Med 40(6):611-618

33. Dillard AJ, Scherer L, Ubel PA, Smith DM, Zikmund-Fisher BJ, McClure JB, Greene S, Stark A, Fagerlin A (2013) Breast cancer anxiety's associations with responses to a chemoprevention decision aid. Soc Sci Med 77:13-19. https://doi.org/10.1016/j.socsc imed.2012.10.009

34. Maheswaran R, Pearson T, Jordan H, Black D (2006) Socioeconomic deprivation, travel distance, location of service, and uptake of breast cancer screening in North Derbyshire, UK. J Epidemiol Community Health 60(3):208. https://doi.org/10.1136/jech.200X. 038398

35. von Wagner C, Baio G, Raine R, Snowball J, Morris S, Atkin W, Obichere A, Handley G, Logan RF, Rainbow S, Smith S, Halloran S, Wardle J (2011) Inequalities in participation in an organized national colorectal cancer screening programme: results from the first 2.6 million invitations in England. Int J Epidemiol 40(3):712718. https://doi.org/10.1093/ije/dyr008

36. Ejiogu N, Norbeck JH, Mason MA, Cromwell BC, Zonderman AB, Evans MK (2011) Recruitment and retention strategies for minority or poor clinical research participants: lessons from the healthy aging in neighborhoods of diversity across the life span study. Gerontologist 51(S1):S33-S45. https://doi.org/10.1093/ geront/gnr027

Publisher's Note Springer Nature remains neutral with regard to jurisdictional claims in published maps and institutional affiliations. 